\title{
Why not take one for a spin?
}

\section{The latest in centrifuges — loads of $g$, masses of speed, but not much noise.}

Genofuge

Techne

www.techne.co.uk

Hush-hush high-speed centrifugation

The Genofuge microcentrifuge is powered bya maintenance-free brushless motor, claimed to give extremely quiet running, and to generate a minimal amount of heat. The unit features a ducting system for a continuous flow of ambient air to keep the rotor and sample cool. For below ambient conditions Genofuge can be used in refrigerated environments. Speed varies from 1,000 to 14,000 r.p.m. to suit microfiltration, separation of proteins and nucleic acids, and other applications.

Reader Service No. 100

K Prima-15R

Composite Rotor Inc. www.composite.com A new angle on carbon fibre

The KOMPspin Prima-15R refrigerated tabletop centrifuge is built for large capacity and quiet running. The unit spins $1.5 \mathrm{ml}, 50 \mathrm{ml}$, and $85 \mathrm{ml}$ tubes at up 15,000 r.p.m. $(27,0000 \mathrm{~g})$ with its non-corroding carbon fibre angle rotor, $1-100 \mathrm{ml}$ and $15 / 50 \mathrm{ml}$ conical tubes in swinging buckets, a four-place 96-well microtitre plates or six-place slides in the cyto rotor. Power is by brushless induction drive.

Reader Service No. 101

\section{Clear-Spin}

Seward

www.seward.co.uk

Here's one to look into

The Clear-Spin microcentrifuge is intended to simplify the manipulation of hazardous samples. The rotor disc doubles as a tube rack, so sample preparation can be performed while the tubes remain in the rotor. The rotor case is hermetically sealed and made from transpar-

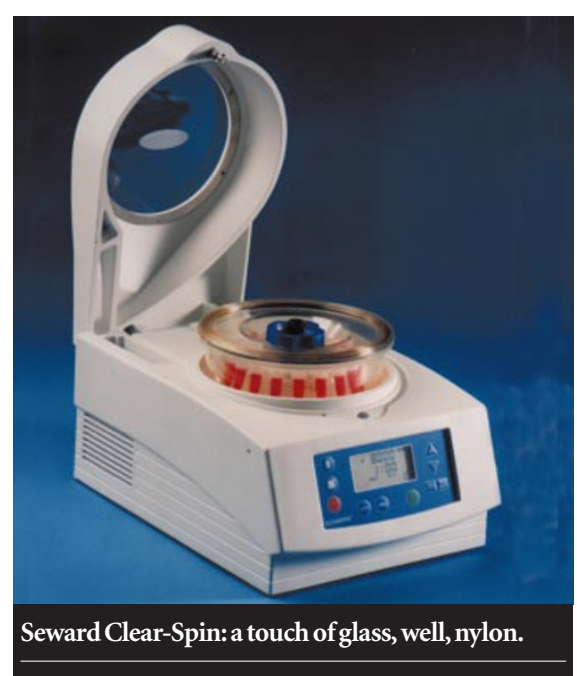

ent nylon so that any spillage can be spotted before lab personnel are exposed to risk. Reader Service No. 102

\section{Centrifuge 5417 C}

Eppendorf www.eppendorf.com

Running hot and cold...

The Eppendorf 5417 C, and the refrigerated $5417 \mathrm{R}$, now have an embossed foil keypad and an extended range of rotors. Microcentrifuge tubes spin-up to $25,000 \mathrm{~g}$ almost instantly in a fixed-angle rotor, and a PCR rotor accommodates eight PCR tube strips. Other rotors supplied are the familiar 30-position fixed-angle rotor for 1.5/2.0 ml tubes (also available aerosol-tight) and a swing-bucket rotor.

Reader Service No. 103

\section{Centra CL3}

IEC www.labcentrifuge.com

Wait for it....

CL3 Series centrifuges are available in refrigerated and non-refrigerated versions. Recent refinements include a 99-protocol storage facility, speed setting to the nearest 10 r.p.m. and automatic calculation of $g$ force. The "at speed timer" assures that the timed run commences only when the rotor reaches the required $g$ force The CL3 accommodates a variety of tubes including $15 \mathrm{ml}, 50 \mathrm{ml}, 225 \mathrm{ml}$ conical tubes, microtubes, microplates microscope slides and filtration devices.

Reader Service No. 104

\section{KR4.22 centrifuge}

Jouan www.jouaninc.com A calculating centrifuge

The Jouan KR 4.22 combines high capacity and high centrifugal force. It can process large numbers of microtitration plates and columns at above $5,000 g$. The proven high torque direct-drive motor is unchanged in the latest models. A digital control system allows the user to control centrifugation precisely. First the centrifuge calculates the total separating force ( $g$-time) applied to samples in a model run, then the run can be regulated to this value. The results can be transferred electronically to Jouan's data acquisition system.

Reader Service No. 105

\section{TJ-25 benchtop}

Beckman Coulter, Inc. www.beckman.com A 'personal' centrifuge for general lab use

Standard equipment on the TJ-25 high-speed refrigerated benchtop centrifuge includes a high- $g$-force $(5,520 g)$, high-volume, swingingbucket TS-5.1-500 rotor, taking four 500-ml samples. This new rotor accommodates adapters for a range of tubes, including $50 \mathrm{ml}$ and $15 \mathrm{ml}$ conical tubes. An optional microtitre plate system for this rotor allows the use of up to four deep-well plates or eight standard plates. An optional biosafety lid for the rotor facilitates biocontainment.

Reader Service No. 106

\section{Hereaus Multifuge}

Kendro Laboratory Products www.kendro.com Thinkbig

Designed for high sample throughput, the Heraeus Multifuge has a tube capacity that the makers claim is the largest in its class. A swinging bucket rotor and a new windshield rotor offer tube capacity up to $4 \times 1,000 \mathrm{ml}$ and $7,000 \mathrm{~g}$. A wide range of adapters offers compatibility with most common tube types. Reader Service No. 107

These notes are compiled in the Nature office from information provided by the manufacturers.

\section{ADVERTISEMENTS}

\section{Custom Peptides \& Polyclonal Antibodies}

Please contact us about complete services for your custom peptide and polyclonal antibody needs.

\section{Research Genetics}

U.S. or Canada 800-533-4363 FAX 256-536-9016

\section{Check out our homepage at} http://www.resgen.com

READER ENQUIRY NO. 39

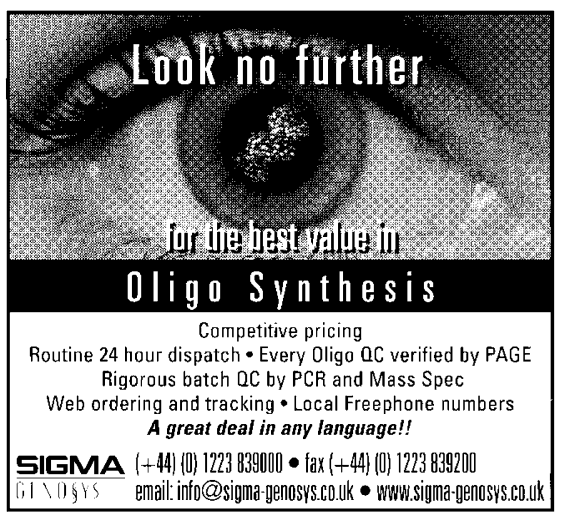

READER ENQUIRY NO. 33 